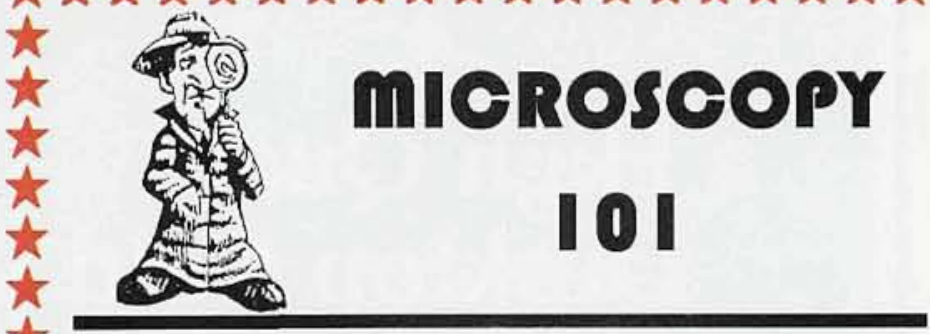

We appreciate the response to this publication feature - and welcome all contributions. Contributions may be sent to Phil Oshel, our Technical Editor at:

\section{Mr. Phil Oshel \\ Station A}

PO Box 5037

Champaign IL 61825-5037

or by eMail: oshe!@ux1.cso.uiuc.edu

\section{Preparing Pellets of Isolated Cells for Frozen Thin-Sectioning:}

We add paraformaldehyde to the cultured monolayers, swirl for just a few minutes (about 5), scrape with a rubber policeman and pellet into a small tipped tube. We use Sarstedt \#72.702. It wouldn't have to be Sarstedt, if somebody else makes them. They just need to have a very small bottom if the pellet is small. These are about $45 \mathrm{~mm}$ long, have an inner diameter of $4 \mathrm{~mm}$, and a smaller tip, shaped like a nipple, the inside diameter of which is about $1 \mathrm{~mm}$. The nipple part is about $7 \mathrm{~mm}$ long.

We pellet in a swinging bucket centrifuge and then microfuge to pack the cells.

We then let them fix for another hour or two and cut off the very bottom and again just above the cells, forming a log with the cells In the center that can be pushed out with a paper clip. If they stick together, fine, proceed.

If not, push them into small piles (about 0.5-1 mm) on a piece of Parafllm, drain them with filter paper cut into pie-shaped wedges using the very tip to touch the pellet gently, and coat them with cooled, still molten $1 \%$ agar. Cut away any excess agar.

Infiltrate with 3 changes of sucrose (2.3M) over about $30-60$ minutes. Place onto stubs and flash freeze. This keeps the cells together, not dispersed thinly in the sucrose.

If the cells are fixed very long before pelleting, they will not like to stick together, and will disperse in the sucrose. The consistency of the cell pellet should be like cooked oatmeal. (I could make some "snotty" comment about consistencies of other substances). If they are too wet, they will disperse. and you'll have to hunt all over your grid for them. If they're too dry, the ultrastructure could be altered

Sara E. Miller, Duke University Medical Center

\title{
Making a Monolayer of Latex Spheres for Calibrating a Scanning Probe Microscope:
}

A z-standard for calibrating a scanning probe microscope made be made by adsorbing latex particles onto a mica surface. The latex must be positively charged, such as amidine-latex particles, since the mica surface is negatively charged in aqueous solution. A very uniform layer of spheres may be obtained just by putting the mica in the latex solution for about an hour. The surface coverage can be adjusted by changing the ionic strength of the solution. Similarly you can also adsorb negatively charged latex particles such as



EM sites often require supplemental magnetic shielding to achieve full resolution. LINEAR RESEARCH ASSOCIATES wideband EMFC-AC electronic active-shielding systems dramatically reduce magnetic fields radiating from nearby a.c. power wiring, ground loops, transformers and related sources. EMFC-QDC extended-range models additionally suppress lowfrequency magnetic disturbances caused by trains, subways, elevators and equipment such as MRI scanners.

Linear Research Associates' EMFC equipment is the highestperformance commercially available magnetic field compensation apparatus in the world. EMFC systems feature state-of-the-art engineeringtt and are U.S.-manufactured to exacting standards. Superior performance and reliability are guaranteed!

Call or fax LRA for complete EMFC-series information. We will also gladly assist with any questions you may have regarding site survey, engineering or EMF issues.

\section{LINEAR RESEARCH ASSOCIATES}

5244 Perry City Road • Trumansburg, NY 14886

Phone (607) 387-3411 • Fax (607) 387-7806

info@linres.com • http://www.linres.com

tt U.S. Patents 5,465,012; 5,469,058

\section{Modern Coating Solutions for Today's EM Sample Prep Needs}

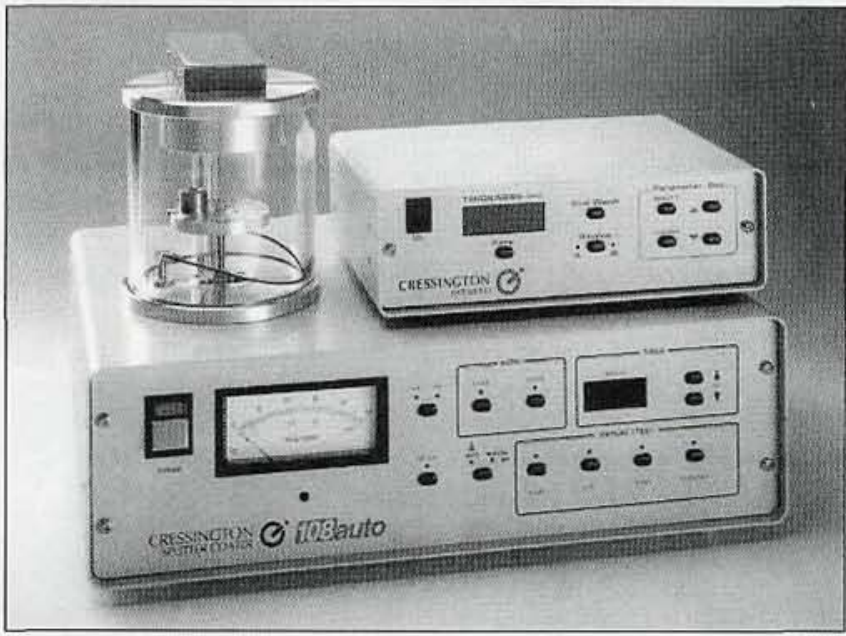

Compact modern desktop systems with fast cycle times. Carbon and sputtering systems for all SEM, FE-SEM and TEM applications.

\section{$\Rightarrow$ CRESSINGTON}

Cressington Scientific Instruments, Inc.

508 Thomson Park Drive, Cranberry Twp., PA 16066 TEL 800-755-1781 / 412-772-0220 FAX 412-772-0219 Website: www.cressington.com 